\title{
LOS DERECHOS DIGITALES DE LOS OBLIGADOS TRIBUTARIOS
}

\author{
María Amparo Grau Ruiz \\ Catedrática de Derecho Financiero y Tributario \\ Universidad Complutense de Madrid (España) \\ Directora de la Revista Técnica Tributaria
}

\author{
Al hilo de la consulta pública \\ recientemente celebrada ${ }^{1}$ sobre la \\ propuesta de una Carta de derechos \\ digitales ${ }^{2}$ de la ciudadanía \\ -conforme a uno de los compromisos \\ fundamentales del plan España \\ Digital 2025-, es oportuno \\ preguntarse si en ella, por su objetivo \\ descriptivo, prospectivo y \\ prescriptivo, tendría sentido incluir \\ algún pronunciamiento expreso
}

-siquiera escueto- sobre tales derechos en el ámbito tributario.

Claramente, a nuestro juicio, abierta la puerta a sugerencias ${ }^{3}$, sí sería pertinente hacer una mínima alusión a esta materia, porque como en tantas otras, se depende, cada día más, de entornos, dispositivos y servicios digitales para relacionarse, comunicarse y realizar actividades.

1 CONSULTA PÚBLICA PARA LA ELABORACIÓN DE UNA CARTA DE DERECHOS DIGITALES. Disponible en el siguiente enlace: https://www.mineco.gob.es/stfls/mineco/ministerio/ participacion_publica/consulta/ficheros/CartaDerechosDigitales.pdf [último acceso: 10 de diciembre de 2020].

2 Propuesta de Carta de derechos digitales, elaborada por el Grupo de Expertos constituido por la Secretaría de Estado de Digitalización e Inteligencia Artificial (SEDIA) del Ministerio de Asuntos Económicos y Transformación Digital. En ella se aclara que por «entorno digital» se entiende el conjunto de sistemas, aparatos, dispositivos, plataformas e infraestructuras que abren espacios de relación, comunicación, interrelación, comercio, negociación, entretenimiento y creación que permiten a las personas físicas o jurídicas de forma bilateral o multilateral establecer relaciones semejantes a los existentes en el mundo físico tradicional. «Espacio digital» se refiere a los lugares digitales que abren los entornos digitales en los que es posible la comunicación, interrelación, comercio, negociación, entretenimiento y creación de forma especular con el mundo físico tradicional. La «ciudadanía digital» se refiere al estatuto de derechos y obligaciones de la persona, con independencia de su estatuto jurídico de nacional.

3 Una vez recogidas las observaciones y aportaciones que se hagan en esta fase de consulta pública, se elaborará el texto final de la Carta de Derechos Digitales, que inspirará los futuros pasos a seguir en la protección de la sociedad de cara a los nuevos retos digitales, fijando los principios sobre los que asentar la salvaguarda de los derechos fundamentales en el escenario digital. España busca colocarse a la vanguardia de la protección de los derechos digitales, tanto por el contenido de la Carta como por su elaboración participativa. https://www.lamoncloa.gob.es/serviciosdeprensa/ notasprensa/asuntos-economicos/Paginas/2020/171120-derechos_digitales.aspx https:// portal.mineco.gob.es/es-es/ministerio/participacionpublica/audienciapublica/Paginas/ SEDIA_Carta_Derechos_Digitales.aspx [último acceso: 10 de diciembre de 2020]. 
Desde un punto de vista sectorial, es importante garantizar que los derechos de los que se disfruta fuera de línea sean también respetados en línea. Por este motivo, es útil tratar de enlazar esta propuesta de Carta con otra medida muy esperada en la Unión europea que afectará directamente a los contribuyentes en un horizonte cercano. Precisamente, entre las iniciativas fiscales que forman parte del Plan de Acción para una tributación justa y sencilla que apoye la recuperación prevista para 2020-2023 se encuentra otra Carta, la de los derechos del contribuyente (en concreto, como Acción A17).

Encuadrada en la citada acción, en el tercer trimestre de 2021 está prevista la aparición de una Comunicación de la Comisión Europea al Parlamento y al Consejo haciendo balance de los derechos de los contribuyentes existentes según el Derecho de la Unión Europea, así como una Recomendación de la Comisión dirigida a los Estados miembros para facilitar la aplicación de los derechos de los contribuyentes y simplificar las obligaciones tributarias ${ }^{4}$. En conexión con este último objetivo, es obviamente muy probable que se deba considerar el uso actual y futuro de las tecnologías digitales.

Como bien se explica en la introducción a la propuesta española de Carta de derechos digitales: «las tecnologías digitales o basadas en lo digital plantean la necesidad de asegurar que el marco normativo garantiza la protección de los derechos individuales y colectivos de las personas». Por este motivo, la Carta pretende concretar los más relevantes en el entorno y los espacios digitales o describir derechos instrumentales o auxiliares de los primeros, a sabiendas que este proceso ha de ser naturalmente dinámico ${ }^{5}$. Asimismo, hace patente su propósito de servir de marco de referencia para la acción de los poderes públicos en lo relacionado con dichas tecnologías, «aprovechando y desarrollando todas sus potencialidades y oportunidades y conjurando sus riesgos». A estas alturas, es bien sabido que el uso de la inteligencia artificial puede afectar a los valores sobre los que se fundamenta la UE y provocar la

4 ANNEX to the COMMUNICATION FROM THE COMMISSION TO THE EUROPEAN PARLIAMENT AND THE COUNCIL AN ACTION PLAN FOR FAIR AND SIMPLE TAXATION SUPPORTING THE RECOVERY STRATEGY, COM (2020) 312 final, Bruselas, 15 de julio de 2020.

5 Como pone de relieve el recorrido desde el Título X de la Ley Orgánica 3/2018, de 5 de diciembre, de Protección de Datos Personales y garantía de los derechos digitales, hasta el Real Decreto-ley 28/2020, de 22 de septiembre, de trabajo a distancia. En particular, en lo fiscal, sobre el teletrabajo cabe destacar que se han hecho interesantes propuestas recientemente: «regular o clarificar la inexistencia de renta en especie en los casos de cesión de uso de todos los medios, equipos y herramientas necesarios para el desarrollo del trabajo a distancia y, la no sujeción de la compensación de gastos, o si se prefiere, la fijación de un umbral de exención a tanto alzado. Resuelto el tema en el IRPF, resuelto, también, el del IVA». DURÁN-SINDREU BUXADÉ, A.: «¿Preocupa de verdad la fiscalidad del teletrabajo?», Cartas a Taxlandia, 17 de noviembre de 2020. https:// www.politicafiscal.es/antonio-duran-sindreu/preocupa-de-verdad-la-fiscalidad-del-teletrabajo 
conculcación de derechos fundamentales ${ }^{6}$.

$\mathrm{Al}$ respecto, en relación con la Carta propuesta, el Consejo General de la Abogacía ha subrayado que «es necesario, desde el punto de vista jurídico, conocer y utilizar las herramientas jurídicas que ya están a nuestro alcance para poder regular con todas las garantías jurídicas y de seguridad este nuevo entorno que nos rodea; evaluaciones de impacto, análisis de riesgos jurídicos y técnicos...» ${ }^{7}$.

Desde luego, la apuesta por una digitalización humanista, que ponga a las personas en el centro, es ciertamente coherente con la propugnada visión antropocéntrica de la inteligencia artificial en el marco de la Unión Europea ${ }^{8}$. En este punto, los derechos de una misma persona pueden ser considerados en paralelo en función de los distintos tipos de relaciones jurídico-económicas en los que se ve inmersa. Así, por ejemplo, la OIT pone en el centro de las tecnologías al trabajador; y, a su vez, las grandes empresas digitales, reinterpretan esta orientación hacia la persona, entendiéndola básicamente como cliente o usuario. Estas variadas perspectivas llevan a pensar que la evolución digital tributaria podría y debería hacerse gravitar, en parecidos términos, en torno al contribuyente.

\section{De conformidad con las} prioridades políticas de la actual Presidenta de la Comisión Europea, un marco regulador claro para Europa generaría confianza entre los 6 Según el Consejo de Europa, un gran número de derechos fundamentales podría verse afectado
por el uso de la IA (https://rm.coe.int/algorithms-and-human-rights-en-rev/16807956b5). Estos riesgos
pueden ser resultado de defectos en el diseño general de los sistemas de IA (especialmente en lo que
se refiere a la supervisión humana) o del uso de datos que puedan ser sesgados sin una corrección
previa (por ejemplo, se entrena un sistema utilizando única o principalmente datos relativos a
hombres, y ello se traduce en resultados peores con relación a las mujeres). Libro Blanco sobre la
inteligencia artificial: un enfoque europeo orientado a la excelencia y la confianza, COM (2020) 65
FINAL, Bruselas, 19 de febrero de 2020, p. 13 (en lo sucesivo, Libro Blanco). Algunos de los riesgos en
materia tributaria ya los pusimos de manifiesto en un editorial anterior. GRAU RUIZ, M.A. (2020)
«Riesgos y oportunidades en la creciente digitalización fiscal», Revista técnica tributaria, No. 130, pp.
7-15.

7 «Desde la Abogacía institucional, entendemos que este avance digital no puede ir separado de la regulación jurídica necesaria que garantice que los derechos fundamentales y las libertades públicas amparados en nuestra Carta Magna estén garantizados en el entorno digital. [...] Resulta de vital importancia que el legislador pondere las consecuencias jurídicas y éticas de la regulación de los denominados «derechos digitales», sin obstaculizar con ello la innovación». https://www.abogacia.es/ wp-content/uploads/2020/O7/OBSERVACIONES-CGAE-CONSULTA-PUBLICA-CARTA-DE-

DERECHOS-DIGITALES.pdf [último acceso: 10 de diciembre de 2020].

8 La Comisión respalda firmemente un enfoque antropocéntrico que se base en la Comunicación Generar confianza en la inteligencia artificial centrada en el ser humano COM (2019) 168, recordada en el documento COM (2020) 65 FINAL. Por su parte, el Parlamento Europeo adoptó en octubre de 2020 tres resoluciones. La primera resolución legislativa de Iban García del Blanco (S\&D, ES) pide a la Comisión que establezca un marco legal europeo completo y preparado para el futuro de principios éticos para el desarrollo, despliegue y uso de la IA, la robótica y las tecnologías relacionadas -incluyendo software, algoritmos y datos- en la Unión. En junio de 2020 se creó un comité especial sobre la inteligencia artificial en la era digital para analizar el futuro impacto de la IA, investigar el reto que supone el despliegue de la IA, analizar el enfoque de terceros países y presentar a las comisiones permanentes responsables del Parlamento una evaluación que defina los objetivos comunes de la UE en esta materia. https://www.europarl.europa.eu/news/en/headlines/society/ 20201015STO89417/ai-rules-what-the-european-parliament-wants [último acceso: 10 de diciembre de 2020]. 
consumidores y las empresas en relación con la inteligencia artificial (en lo sucesivo, IA) y, por consiguiente, aceleraría su adopción. Esta visión pragmática no olvida que dicho marco debe garantizar resultados óptimos desde el punto de vista social, medioambiental y económico, así como su conformidad con la legislación, los principios y los valores de la UE. Y se es perfectamente consciente de que ello resulta especialmente relevante en sectores en los que los derechos de los ciudadanos se ven afectados de manera más directa; por ejemplo, en el caso de las aplicaciones de IA empleadas por los cuerpos y fuerzas de seguridad y el poder judicial ${ }^{9}$. En esta línea de razonamiento, cabe aducir que otra área en la que todos los ciudadanos se ven seriamente afectados en sus derechos de manera cotidiana es, sin duda, la tributaria.

Por supuesto, la Carta propuesta en España ya contiene referencias genéricas a la administración pública y a los procesos de transformación digital que ésta afronta. En ese marco, puede entenderse que tiene cabida lo tributario. Literalmente se dice [la negrita es nuestra]:

XVI Derechos digitales de la ciudadanía en sus relaciones con las Administraciones públicas

1. Se reconoce el derecho de igualdad en el acceso a los servicios públicos y en las relaciones digitales con las Administraciones públicas. A tal fin se promoverán políticas públicas activas que garanticen el acceso a los sistemas y los procedimientos.
2. El poder público autor de una actividad en el entorno digital deberá identificar a los órganos responsables de la misma.

3. El principio de transparencia y de reutilización de datos de las Administraciones públicas guiará la actuación de la Administración digital, de conformidad con la normativa sectorial. En particular, se garantizará el derecho de acceso a la información pública, se promoverá la publicidad activa y la rendición de cuentas y se velará por la portabilidad de los datos y la interoperabilidad de los formatos, sistemas y aplicaciones.

4. Siempre que sea posible se promoverá la universalidad y la neutralidad de las tecnologías usadas por las Administraciones públicas, así como su diseño y uso conforme a los principios éticos que acompañan a esta Carta. Así mismo se adoptarán las medidas precisas para garantizar que la prestación de los proveedores de servicios que colaboren con ellos por medios digitales se realicen conforme a las disposiciones de esta Carta.

5. Se ofrecerán alternativas en el mundo físico que garanticen los derechos de aquellas personas que opten por no utilizar recursos digitales.

6. Los daños causados por actividades o decisiones digitales, podrán dar lugar a un derecho a la indemnización por toda lesión que las personas físicas o jurídicas sufran en cualquiera de sus bienes $y$ derechos, de acuerdo con las leyes

9 Libro Blanco, p. 12. 
7. Los derechos de la ciudadanía en relación con la Inteligencia Artificial reconocidos en esta Carta $\left.{ }^{[10}\right]$ resultarán también de aplicación en el marco de la actuación administrativa, en particular en los aspectos referidos al diseño y al uso de algoritmos. En todo caso, se reconoce el derecho a: a) Que las decisiones y actividades en el entorno digital respeten los principios de buen gobierno y el derecho a una buena Administración digital. b) Un procedimiento de toma de decisiones con las debidas garantías. c) Obtener una motivación comprensible en lenguaje natural de las decisiones que se adopten en el entorno digital, con justificación de las normas jurídicas relevantes al caso y de los criterios de aplicación de las mismas d) Que la adopción de decisiones discrecionales quede reservada a personas, salvo que una norma con rango de ley permita la adopción de decisiones automatizadas en este ámbito. Será necesaria una evaluación de impacto en los derechos digitales en el diseño de los algoritmos en el caso de adopción de decisiones automatizadas $o$ semiautomatizadas. En todo caso, serán objeto de aprobación previa de los sistemas algorítmicos que se vayan a usar para la toma de decisiones, con determinación de su ámbito concreto de aplicación y estructura de funcionamiento.

En ocasiones, otros derechos a los que se hace mención en esta Carta de derechos digitales también podrán encontrar reflejo indirecto en cuestiones tributarias. Por ejemplo, el derecho a no ser localizado y perfilado, cuya incorporación expresa se propone en el apartado $\mathrm{V}^{11}$.

En el campo tributario, no está de más que, tras la apresurada digitalización de los procedimientos, llegue la hora de digitalizar también, de algún modo, los derechos de los contribuyentes. Esto es, de concretarlos adecuadamente para que se puedan ejercer con igual o mayor eficacia en el mundo digital, proyectando el Ordenamiento vigente y sus posibles mejoras sobre la realidad tecnológica, de manera que

10 XXIII Derechos ante la Inteligencia artificial

1. En el desarrollo y ciclo de vida de los sistemas de Inteligencia Artificial: a) Se deberá garantizar el derecho a la no discriminación algorítmica, cualquiera que fuera su origen, causa o naturaleza del sesgo, en relación con las decisiones y procesos basados en algoritmos. b) Se asegurarán la transparencia, auditabilidad, explicabilidad y trazabilidad. c) Deberán garantizarse la accesibilidad, usabilidad y fiabilidad.

2. Las personas tienen derecho a no ser objeto de una decisión basada únicamente en procesos de decisión automatizada, incluidas aquéllas que empleen procedimientos de inteligencia artificial, que produzcan efectos jurídicos o les afecten significativamente de modo similar, salvo en los supuestos previstos en las leyes. En tales casos se reconocen los derechos a: a) Solicitar una supervisión e intervención humana; b) Impugnar las decisiones automatizadas o algorítmicas.

3. Se deberá informar a las personas sobre el uso de sistemas de Inteligencia Artificial que se comuniquen con seres humanos utilizando el lenguaje natural en todas sus formas. Deberá garantizarse en todo caso la asistencia por un ser humano a solicitud de la persona interesada.

4. Se prohíbe el uso de sistemas de Inteligencia Artificial dirigidos a manipular o perturbar la voluntad de las personas, en cualesquiera aspectos que afecten a los derechos fundamentales.

11 1. El derecho a la libre autodeterminación individual y la garantía de las libertades comporta el derecho a no ser objeto de localización, ni a ser sometido a análisis de la personalidad o conducta que impliquen el perfilado de la persona. 2. Sólo serán posibles tales tratamientos de información personal con el consentimiento de la persona afectada o en los casos y con las garantías previstos en las leyes. 
una interpretación sistemática promueva además la seguridad jurídica. En esta línea se han alzado voces autorizadas ${ }^{12}$.

Para asegurar prácticas de tributación justa, la Comisión recomienda simplificar las obligaciones tributarias y respetar a los derechos de los contribuyentes. Una de las vías a seguir es identificar las buenas prácticas administrativas y mejorar la coordinación de las normas nacionales ${ }^{13}$. En la pretensión de optimizar las relaciones de los contribuyentes con las Administraciones tributarias, es acertado fomentar el uso de las tecnologías digitales de manera coordinada a escala de la Unión Europea, sin olvidar el papel fundamental de los asesores fiscales.

La Confederación Fiscal Europea, sabedora de que «los programas de cumplimiento cooperativo, la transparencia fiscal y el cumplimiento de los impuestos en general se facilitan enormemente con la automatización y la digitalización de los procesos», ha entendido «que la
Comisión Europea tiene la intención de investigar herramientas y soluciones digitales, en particular para mejorar la capacidad de análisis de datos de las administraciones fiscales y pasar del intercambio de información a un modelo en el que los datos fiscales puedan compartirse en tiempo real». No obstante, ha señalado «las deficiencias de los recursos digitales de algunas jurisdicciones, que obstaculizan la capacidad de los ciudadanos y los contribuyentes para acceder a los servicios y cumplir sus obligaciones de presentación de informes y otras obligaciones». A su juicio, «la inversión en tecnología de la información beneficiaría a las empresas y a las autoridades fiscales al mejorar la eficiencia y la calidad de los datos, la comunicación y el acceso remoto a los servicios» ${ }^{14}$.

Cabe recordar que el modelo de Carta del Contribuyente publicado hace años por la Confederación Fiscal Europea ya contenía ejemplos de lo que un buen sistema tributario debería incluir sobre la base de las obligaciones con respecto al

\footnotetext{
12 En la Conferencia de Asuntos profesionales de los Asesores fiscales de la CFE, el pasado 30 de noviembre, se abordó el tema de «los derechos de los contribuyentes y la seguridad jurídica en la era digital», analizándose los desarrollos a nivel europeo y mundial en la protección de los mismos y las implicaciones que las nuevas tecnologías tienen en ellos.

13 https://ec.europa.eu/info/law/better-regulation/have-your-say/initiatives/12627-EU-taxpayersrights-simplified-procedures-for-better-tax-compliance-Recommendation- [último acceso: 10 de diciembre de 2020].

14 Algunos programas de cumplimiento cooperativo (como el de los Estados Unidos) están supeditados a la presentación de informes en tiempo real sobre las transacciones contables y fiscales, y esto sólo es posible cuando tanto las autoridades fiscales como los contribuyentes invierten en soluciones de tecnología de la información. «European tax professionals call for better tax governance», Joint statement of Accountancy Europe and CFE Tax Advisers Europe, 10 de julio de 2020. Disponible en el enlace https://www.accountancyeurope.eu/tax/european-taxprofessionals-call-for-better-tax-governance/ [último acceso: 10 de diciembre de 2020].
} 
Estado $^{15}$. En 2016 la Confederación apoyó las directrices de la Comisión (Guidelines for a Model for a European Taxpayers' Code) al reconocer que este tipo de Código o Carta puede mejorar la eficiencia y la eficacia del sistema tributario, dejando a los Estados la elección sobre el enfoque normativo a adoptar.

En la actualidad, de cara a elaborar la futura recomendación para mejorar la situación de los contribuyentes en el mercado de la Unión Europea, la hoja de ruta presentada justifica la actuación de la Comisión con base en los siguientes argumentos: «El desconocimiento y la utilización no óptima de los derechos de los contribuyentes existentes pueden tener un efecto negativo en el comportamiento económico y empresarial. Al afectar a los contribuyentes que tienen actividades o intereses transfronterizos, esto puede obstaculizar el buen funcionamiento y el pleno potencial del mercado único. El aumento del conocimiento de los derechos de los contribuyentes puede contribuir a suavizar la relación entre los contribuyentes y las administraciones tributarias, lo cual es particularmente importante en el contexto de la recuperación. También puede mejorar, como consecuencia natural, el cumplimiento de las obligaciones fiscales» ${ }^{16}$.
En definitiva, vale la pena recalcar que conviene a las propias administraciones tributarias, no sólo conocer, sino también utilizar los derechos de los contribuyentes, para no frenar la actividad económica. Aunque se aprecien ciertos tintes mercantilistas en el enfoque, por el bien de los obligados tributarios, es de agradecer este recordatorio. Por otro lado, se añade que el mejor uso de los avances tecnológicos puede desembocar en una recaudación de tributos más sencilla, más eficaz y fácil, también en situaciones transfronterizas, mientras que se consigue la justicia social. Habrá que seguir muy de cerca en el despliegue de estas tecnologías, sus costes de financiación y la necesaria coordinación que evite excesivos costes de cumplimiento.

Otras cuestiones contempladas en la Carta propuesta de derechos digitales, que interesan en el ámbito fiscal, son la relativa al derecho a la participación ciudadana por medios digitales (XIV) y la garantía de los derechos en los entornos digitales (XXV). Conforme a la primera, se promoverán entornos digitales que contribuyan a un derecho de acceso efectivo a la información pública, la transparencia y la rendición de cuentas. De acuerdo con la segunda, sin perjuicio de lo dispuesto en la legislación sectorial específica, todas

\footnotetext{
15 CFE Professional Affairs Committee: Opinion Statement PAC 4/2020 on the European Commission initiative/ roadmap for Communication and Recommendation to Improve the Situation of Taxpayers in the Single Market of 31 October 2020. Enviado a las instituciones europeas el 26 de noviembre de 2020. Michael Cadesky, Ian Hayes, David Russell (2016) «Towards Greater Fairness in Taxation: A Model Taxpayer Charter», CFE \& IBFD. https://taxadviserseurope.org/blog/portfolioitems/the-model-taxpayer-charter/ [último acceso: 10 de diciembre de 2020]. CFE Survey on Taxpayers Charters (2019) https://taxadviserseurope.org/blog/cfe-tax-advisers-europe-and-gtapleading-the-work-on-taxpayer-charters-and-rights/ [último acceso: 10 de diciembre de 2020]. VALENTE, P. (2017) «A European Taxpayers» Code», Intertax, Vol. 45, No. 12, pp. 807-815.

16 Comisión Europea: Roadmap -Title of the initiative: «Recommendation to Improve the Situation of Taxpayers in the Single Market», Ref. Ares(2020)6140272-30 de octubre de 2020. Como guardiana de los Tratados, «la Comisión es la más indicada para recomendar la forma de mejorar los derechos de los contribuyentes en virtud de la legislación de la Unión Europea en el Mercado Único». Se apoya en el artículo 17 del Tratado de la Unión Europea y el artículo 292 TFUE.
} 
las personas tienen derecho a la tutela administrativa y judicial de sus derechos en los entornos digitales. También se promoverán mecanismos de autorregulación «regulada» y procedimientos de resolución alternativa de conflictos.

De hecho, en cuanto a la transparencia, la rendición de cuentas y la conveniencia de evitar la conflictividad en los tiempos que corren, cabe mencionar el ejemplo de un estudio reciente sobre el deseable comportamiento en la Agencia tributaria canadiense (Canadian Revenue Agency, CRA) -que cabría igualmente esperar en España ante la crítica situación empresarial tras la pandemia ${ }^{17}$-. En él se resalta la idea de que, más allá de la importante inversión en mejoras tecnológicas dirigidas a mejorar la eficiencia de las inspecciones en la CRA, los gobiernos deben resistir el impulso de financiar las medidas adoptadas con motivo del COVID-19 y la pérdida de ingresos fiscales durante esta crisis pidiendo a la CRA que simplemente obtenga más ingresos a través de un uso agresivo de los procedimientos de aplicación de los tributos. Se agrega: aunque los resultados de la inspección puedan ser objeto de impugnaciones, no es conveniente confiar frecuentemente en apelaciones y tribunales para asegurar que las normas fiscales se apliquen de manera justa ${ }^{18}$.

Así pues, la Administración tributaria para ser confiable, en el fondo y en la forma, necesita en esencia mejorar el estatuto jurídico del contribuyente teniendo en cuidadosamente cuenta los riesgos y oportunidades que plantean para ambas partes los medios digitales y la toma de decisiones a partir de tecnologías de esta naturaleza. La confianza es fundamental en el rumbo europeo, conforme se reconoce en el Libro Blanco sobre la inteligencia artificial, debiendo siempre considerarse el impacto de los sistemas de IA no solo desde una perspectiva individual, sino también desde la perspectiva de la sociedad en su conjunto ${ }^{19}$. Para generar un ecosistema de confianza el marco normativo debe velar por el

17 Como indica Óscar Arce, Director general de Economía y Estadística del Banco de España: «las políticas económicas han contribuido, hasta ahora, a mitigar los riesgos de liquidez de las empresas, si bien la presión sobre la solvencia empresarial constituye uno de los principales retos en el corto y medio plazo». https://www.bde.es/bde/es/-impacto-del-covid-19-sobre-la-situacion-financiera-de-lasempresas-no-financieras-en-2020---central-826274de71a1671.html El retroceso de la rentabilidad habría sido especialmente intenso en el segmento de pymes y, sobre todo, en los sectores más afectados por la crisis. La crisis habría ocasionado un fuerte aumento de la presión financiera soportada por las empresas, así como un deterioro, aunque más moderado, de su solvencia. https:// www.bde.es/f/webbde/GAP/Secciones/SalaPrensa/IntervencionesPublicas/DirectoresGenerales/ economia/Arc/Fic/arce011220.pdf [últimos accesos: 10 de diciembre de 2020].

18 KLASSEN, K.J.; PANTALEO, N. (2020) «Assessing the Canada Revenue Agency: Evidence on Tax Auditors» Incentives and Assessments», C.D. HOWE Institute Trusted Policy Intelligence e-Brief, 3 de septiembre, pp. 4 y 11.

19 Se reconoce explícitamente que el uso de sistemas de inteligencia artificial puede tener un papel importante en la consecución de los Objetivos de Desarrollo Sostenible (ODS) y en el respaldo de los procesos democráticos y los derechos sociales. Libro Blanco, p. 3. Recíprocamente, desde el ámbito de los Informes en los avances en sostenibilidad se vinculan los ODS a la digitalización. Así, en las seis transformaciones prioritarias de los ODS dentro de la UE, se incluye el punto 6 sobre la transformación digital: Construir una infraestructura digital de vanguardia, fortalecer la innovación y proteger los derechos de los ciudadanos a sus datos y a la democracia europea. Esto requerirá inversiones sustanciales en innovación tecnológica e infraestructura digital. SDSN e IEEP (2020) The 2020 Europe Sustainable Development Report: Meeting the Sustainable Development Goals in the face of the COVID-19 pandemic, Sustainable Development Solutions Network e Institute for European Environmental Policy, París y Bruselas, p. vii. 
cumplimiento de las normas de la UE, especialmente las normas de protección de los derechos fundamentales y los derechos de los consumidores, en concreto en relación con los sistemas de inteligencia artificial que operan en la UE y presentan un riesgo elevado. A nuestro juicio, en las circunstancias actuales, este es también el caso de los derechos de los obligados tributarios (expandiendo el análisis de riesgos y la tecnología más allá de su utilización limitada en la lucha contra el fraude fiscal).

La Comisión considera que, en general, una aplicación de IA determinada debe considerarse de riesgo elevado en función de lo que esté en juego, y considerando si tanto el sector como el uso previsto suponen riesgos significativos, en especial desde la perspectiva de la protección de la seguridad, los derechos de los consumidores y los derechos fundamentales. Existe riesgo elevado cuando concurran los dos criterios siguientes: $1^{\circ} \mathrm{La}$ aplicación se emplee en un sector en el que, por las características o actividades que se llevan a cabo normalmente, es previsible que existan riesgos significativos. Por ejemplo, la sanidad, el transporte, la energía y determinados ámbitos del sector público (como la seguridad social y los servicios de empleo). El elenco deberá revisarse periódicamente en función de los desarrollos pertinentes en la práctica. $2^{\circ}$ Se use, además, de manera que puedan surgir riesgos significativos. La evaluación del nivel de riesgo de un uso determinado puede basarse en las repercusiones para las partes afectadas. Por ejemplo, el uso de aplicaciones de IA con efectos jurídicos o similares en los derechos de un particular o de una empresa. Puede haber, además, casos excepcionales en los que, debido a lo que esté en peligro, el uso de aplicaciones de IA para determinados fines se considere de elevado riesgo en sí mismo. Por ejemplo, en vista de su importancia para las personas y del acervo de la UE en materia de igualdad de empleo, el uso de las aplicaciones de IA en los procedimientos de contratación y en situaciones que repercutan en los derechos de los trabajadores debe considerarse siempre de «riesgo elevado» 20 .

En la situación en la que nos encontramos, todo el entorno o espacio tributario, alrededor del contribuyente (y por extensión, los demás obligados), va digitalizándose paulatinamente a ritmo acelerado, por lo que es preciso arbitrar los contrapesos necesarios para que el desarrollo del Derecho tributario en su actualización sea equilibrado. Basta revisar brevemente, para percatarse de la tendencia, las Conclusiones del Consejo ECOFIN de 27 de noviembre de 2020 «sobre una fiscalidad equitativa y eficaz en tiempos de recuperación, sobre los desafíos fiscales vinculados a la digitalización y sobre la buena gobernanza en el ámbito fiscal en la

20 Libro Blanco, p.17. 
UE y fuera de ella» ${ }^{21}$. En el IVA apoya seguir ampliando la facturación electrónica; estudiar la utilización de nuevas tecnologías para mejorar la eficiencia de la presentación de informes y el control de los datos; pasar a la puesta en común automatizada de datos mediante sistemas electrónicos interoperativos y facilitar la armonización y la normalización de los datos en relación con las transacciones transfronterizas... En el apartado VI de estas Conclusiones, dedicado a la Administración tributaria y cumplimiento de las obligaciones fiscales, el ECOFIN respalda tanto la iniciativa prevista de la Comisión sobre los derechos de los contribuyentes como la necesidad de elaborar pautas o mecanismos normalizados de procesamiento de datos a los efectos de la recaudación de ingresos. Es hora de valorar cautelosamente los costes y beneficios económicos, administrativos y sociales que comportan estos procesos para todos los agentes implicados.
En virtud del contexto descrito, la participación pública de entidades asociativas sectoriales, representativas de intereses colectivos -como AEDAF-, sería requerida en para el buen orden de las cosas en los procesos de tranformación digital en curso que afectan a los obligados tributarios en particular y a la sociedad en su conjunto. De ahí que, también la configuración de los mecanismos de vigilancia ex ante y ex post del desarrollo de las tecnologías digitales en lo fiscal deba hacerse con espíritu colaborativo y constructivo. En los centros de innovación digital también podría asesorarse alas PYMES sobre nuevos aspectos formales relacionados con la tributación. $\mathrm{O}$ incluso a la hora de la rendición de cuentas del actuar administrativo, podría permitirse la participación en el control del cumplimiento de los derechos $^{22}$. ¿Por qué no?

21 «41. TOMA NOTA de la iniciativa prevista de la Comisión sobre los derechos de los contribuyentes, CONVIENE en que la forma de una comunicación es una primera medida apropiada de concienciación en esta esfera y PIDE que se lleve a cabo un mayor análisis a este respecto, para facilitar cualquier debate futuro sobre esta cuestión; 42. RECONOCE la necesidad de seguir trabajando para mejorar la eficiencia del control del cumplimiento de las obligaciones tributarias en la UE y, habida cuenta del rápido desarrollo de la tecnología, de elaborar pautas o mecanismos normalizados de procesamiento de datos a los efectos de la recaudación de ingresos y la identificación de los riesgos de fraude fiscal, de modo que puedan aprovecharse al máximo la utilidad y los beneficios de la conservación y el procesamiento de los datos recogidos por las autoridades tributarias». Además, en relación con el IVA, pide a la Comisión que garantice que todas sus posibles propuestas se evalúen plenamente en función de sus costes y beneficios económicos, administrativos y sociales para los contribuyentes y las autoridades fiscales, incluidas sus repercusiones en las capacidades informáticas y en los derechos fundamentales, como la protección de los datos personales (punto 16); RESULTADO DE LOS TRABAJOS (OR. en) 13350/20 FISC 226 ECOFIN 1097, Bruselas, 27 de noviembre de 2020. Disponible en el enlace: https://data.consilium.europa.eu/doc/document/ST-13350-2020-INIT/ es/pdf [último acceso: 10 de diciembre de 2020].

22 Las evaluaciones de conformidad deben ser de obligado cumplimiento para todos los agentes económicos sujetos a los requisitos, independientemente del lugar en que estén establecidos. A fin de limitar la carga para las pymes, puede preverse alguna estructura de apoyo, especialmente mediante los centros de innovación digital. Además, es posible contar con medidas y herramientas especializadas en línea para facilitar el cumplimiento. Toda evaluación previa de la conformidad debe realizarse sin perjuicio de la supervisión del cumplimiento y de la posterior ejecución por parte de las autoridades nacionales competentes. Los controles ex post deben facilitarse mediante una adecuada documentación de la aplicación de IA pertinente y, cuando proceda, ofreciendo la posibilidad de que terceros (como las autoridades competentes) prueben dichas aplicaciones. Ello puede resultar especialmente importante cuando surjan riesgos para los derechos fundamentales que dependan del contexto. Libro Blanco, pp.28-29. 